\title{
Fribrinolytic activity and gas production by Pleurotus ostreatus-IE8 and Fomes fomentarius - EUM1 in bagasse cane
}

\section{Actividad fibrolítica y producción de gas por Pleurotus ostreatus- IE8 y Fomes fomentarius-EUM1 en bagazo de caña}

\author{
Paulino Sánchez-Santillán, ${ }^{1,5}$ Ph.D, Marcos Meneses-Mayo, 2* Ph.D, Luis Miranda-Romero, ${ }^{3}$ Ph.D, \\ Eduardo Santellano-Estrada, ${ }^{4}$ Ph.D, Baldomero Alarcón-Zúñiga, ${ }^{3}$ Ph.D.
}

\begin{abstract}
${ }^{1}$ Colegio de Postgraduados, Campus Montecillo. Carretera México-Texcoco, Km 36.5, Montecillo, Estado de México, México, C.P. 56230. ${ }^{2}$ Universidad Anáhuac México-Norte, Facultad de Ciencias de la Salud (Nutrición), Av. Universidad Anáhuac No 46, Colonia Lomas Anáhuac Huixquilucan, Estado de México, México, C.P. 52786. ${ }^{3}$ Universidad Autónoma Chapingo, Departamento de Zootecnia. Carretera México-Texcoco, Km 38.5, Chapingo, Estado de México, México, C.P. 56230. ${ }^{4}$ Universidad Autónoma de Chihuahua, Facultad de Zootecnia. Periférico Francisco R. Aldama, km 1, Chihuahua, Chihuahua, C.P. 31543. ${ }^{5}$ Universidad Autónoma de Guerrero, Unidad Académica de Medicina Veterinaria y Zootecnia N², Carretera Acapulco Pinotepa Nacional Kilómetro 197, Cuajinicuilapa, Guerrero, México, C.P. 41940 *Correspondence: marcos.meneses@anahuac.mx
\end{abstract}

Received: February 2015; Acepted: May 2015.

\begin{abstract}
Objective. To characterize the fibrolytic enzymatic activity of Pleurotus ostreatus-IE8 and Fomes fomentarius-EUM1 in sugarcane bagasse (BCA); to evaluation of the kinetics of in vitro production of BCA treated by solid fermentation (FS), crude enzyme extract (ECE) of $P$. ostreatus-IE8 and Fibrozyme ${ }^{\circledR}$. Materials and methods. In fungi measured radial growth rate ( $\mathrm{Vcr}$ ) and biomass production in two culture media (with or without nitrogen source); activity of xylanases, cellulases and FS on BCA at 0,7 and $15 \mathrm{~d}$. The chemical analysis and kinetic analysis of in vitro gas production in 4 treatments (ECE adding enzymes obtained from the direct addition FS or FS ), witness (Fibrozyme ${ }^{\circledR}$ ) and a control without addition and analyzed by a was completely randomized design. Results. Xylanases $(7 \mathrm{~d})$ showed 6.32 and $5.50 \mathrm{UI} \mathrm{g}^{-1}$ initial substrate dry weight (SSi) for fungi $P$. ostreatus-IE8 and $F$. fomentarius-EUM1, respectively ; $P$. ostreatus-IE8 scored higher activity of laccases $\left(10.65 \mathrm{~g}^{-1} \mathrm{UI}\right.$ $\mathrm{SSi}$ ) and $F$. fomentarius-EUM1 (1.90 UI g $\left.{ }^{-1} \mathrm{SSi}\right)$ cellulases. The ECE of $P$. ostreatus-IE8 and commercial enzyme did not differences $(p>0.05)$. In the chemical composition or the gas production kinetics. The 4 treatments evaluated decreased values of the variables measured in the kinetics of gas production compared to the control $(\mathrm{p} \leq 0.05)$. Conclusions. The ECE of $P$. ostreatus-IE8 was similar to commercial enzyme degradation in vitro, so it is feasible to use pre-digest high fiber products.
\end{abstract}

Key words: Fibrolytic enzymes, in vitro digestibility, solid fermentation, sugarcane bagasse (Source: DeCS).

\section{RESUMEN}

Objetivos. Caracterizar la actividad enzimática fibrolítica de Pleurotus ostreatus-IE8 y Fomes fomentarius-EUM1 en bagazo de caña de azúcar (BCA) y evaluar la cinética de producción de gas in vitro del BCA por fermentación sólida (FS) o con extractos crudos enzimáticos (ECE) de $P$. ostreatus-IE8 
o Fibrozyme ${ }^{\circledR}$. Materiales y métodos. En los hongos de estudio se evaluó la velocidad de crecimiento radial ( Vcr) y producción de biomasa en dos medios de cultivo (con o sin fuente de nitrógeno); actividad de xilanasas, celulasas y lacasas de la FS sobre BCA a 0,7 y $15 \mathrm{~d}$. El análisis químico y cinética de producción de gas in vitro en 4 tratamientos (proceso de FS o adición de enzimas obtenidas de ECE de la FS), un testigo (Fibrozyme ${ }^{\circledR}$ ) y un control sin adición de enzimas, todo ello se analizó en un diseño completamente al azar. Resultados. Las xilanasas (7 d) mostraron 6.32 y $5.50 \mathrm{UI} \mathrm{g}^{-1}$ sustrato seco inicial (SSi) en $P$. ostreatus-IE8 y $F$. fomentarius-EUM1, respectivamente. $P$. ostreatus-IE8 mostró mayor actividad lacasa (10.65 UI g-1 SSi) y $F$. fomentarius-EUM1 $\left(1.90 \mathrm{UI} \mathrm{g}^{-1} \mathrm{SSi}\right)$ de celulasas. El ECE de $P$. ostreatus-IE8 y Fibrozyme ${ }^{\circledR}$ no presentaron diferencias $(p>0.05)$ en la composición química ni en la cinética de producción de gas. Los 4 tratamientos evaluados disminuyeron los valores de las variables medidas en la cinética de producción de gas in vitro respecto al testigo $(p \leq 0.05)$. Conclusiones. El ECE de $P$. ostreatus-IE8 fue similar a Fibrozyme ${ }^{\circledR}$ en la degradación in vitro, indicando su viabilidad y uso para pre-digerir subproductos altos en fibra.

Palabras clave: Bagazo de caña de azúcar, digestibilidad in vitro, enzimas fibrolíticas, fermentación sólida (Fuente: DeCS).

\section{INTRODUCTION}

The cell wall composition of sugarcane bagasse (SCB) has repercussions in ruminal digestion, considered to be relatively slow and incomplete. The nutritional value of SCB is low, and adding it as an ingredient to feed increases production costs. Biotechnological studies have shown that a combination of physical, chemical and biological treatments increase digestibility and nutritional quality of agro industrial byproducts (including SCB). Results are vary though (1). Okano et al (2) indicates that an option to increase the chemical availability of nutrients and the assimilation of byproducts of lignocellulosic fungi is solid fermentation (SF) and the use of fibrolytic exogenous enzymes, natural to these microorganisms and that are produced through liquid fermentation or in solid state (3).

The physiological and morphological characteristics of filamentous fungi allow for a good production of fibrolytic extracellular enzymes through SSF. Cellulase are enzymes that hydrolyze glyosidic bonds $\beta-1,4$ found in polysaccharides of cellulose. Xylanase acts in synergy and in sequence on the xylan structure to degrade it into constitutive sugar (4), and laccase, are polyphenol oxidase enzymes that oxidize phenolic compounds, the main element in lignin (5). The objective of this research is to characterize the fibrolytic enzymatic activity of a $P$. ostreatus-IE8 and $F$. fomentarius-EUM1culture in sugarcane bagasse; characterize the chemical composition and in vitro fermentation of SCB treated by $\mathrm{SF}$, resulting in CEE of $P$. ostreatusIE8 and to compare it with a commercial extract.

\section{INTRODUCCIÓN}

La composición de la pared celular del bagazo de caña de azúcar (BCA) está implicada directamente en la digestión ruminal, por considerarse relativamente lenta e incompleta. El valor nutritivo del BCA es bajo y su inclusión como ingrediente en dietas aumenta los costos de producción. Estudios biotecnológicos demuestran que una mezcla de tratamientos físicos, químicos y biológicos mejora la digestibilidad y calidad nutritiva de los subproductos agroindustriales (incluido el BCA), pero los resultados son variables (1). Okano et al (2) indican que una alternativa para mejorar la disponibilidad química de sus nutrientes y la asimilación de los subproductos por hongos lignocelulósicos es la fermentación sólida (FS) y el uso de enzimas fibrolíticas exógenas propias de estos microorganismos que producen mediante fermentaciones líquidas o en estado sólido (3).

Las características fisiológicas y morfológicas de los hongos filamentosos permiten una buena producción de enzimas extracelulares fibrolíticas obtenidas por FS. Las celulasas son enzimas que hidrolizan enlaces glucosídicos $\beta-1,4$ en los polisacáridos de la celulosa; las xilanasas actúan de manera sinérgica y secuencial en la estructura del xilano para degradarlo en azúcares constitutivos (4) y las lacasas son enzimas polifenol-oxidasas que oxidan compuestos fenólicos, constituyentes principales de la lignina (5). El objetivo de este estudio fue caracterizar la actividad enzimática fibrolítica del cultivo de los hongos $P$. ostreatus-IE8 y $F$. fomentariusEUM1 en bagazo de caña de azúcar, así como su composición química y fermentación in vitro del BCA tratado por FS, obtención de ECE de $P$. ostreatus-IE8 y su comparativo con un extracto comercial. 


\section{MATERIALS AND METHODS}

Research Site. The investigation was conducted in the Microbiology Laboratory of the Zootechnic Department at the Universidad Autonoma Chapingo, and the Colegio de Posgraduados, both in the State of Mexico.

Inoculation. Two strains of Pleurotus ostreatus-IE8 and Fomes fomentarius-EUM1 were used, both with the capacity to produce lignocellulosic enzymes. The EUM1 strain was taken from cultures available at the Universidad Autonoma Metropolitana and strain IE8 from the Colegio de Posgraduados.

Culture Medium. The strains were reactivated in an agar-malt extract and propagated in an initiating substrate using a mixture of sorghum grain and SCB in a ratio of $80: 20$, to be used later in the SF with SCB.

Experiments. Two experiments were conducted: The first one evaluated the enzymatic activity, radial speed growth ( $\mathrm{rsg}$ ), and biomass of the $P$. ostreatus-IE8 and $F$. fomentarius-EUM1 fungi strain. The second one determined the kinetics of in vitro fermentation using the gas production technique (6) of the SCB treated for $7 \mathrm{~d}$ with SF with the $P$. ostreatus-IE8 strain - evaluation of the CEE for $7 \mathrm{~d}$ with $P$. ostreatus-IE8-. These were compared with a commercial enzyme (Fibrozyme ${ }^{\circledR}$, Alltech Inc., Nicholasville, KY) and a control, as well as the chemical composition of the treatments, controls and study control.

Determining the radial speed growth (rsg) and the dry matter of biomass. The rsg for both strains was measured for $7 d$ in two culture mediums using the Trinci method (7): Medium 1 (M1) with sugarcane bagasse extract (BE) obtained by boiling $100 \mathrm{~g}$ of SCB in $1 \mathrm{~L}$ of distilled water for $30 \mathrm{~min}$. Agar-agar (1.5\%) and caseine peptone $(0.5 \%)$ were added. Medium 2 (M2) had BE and agar-agar $(1.5 \%)$ without caseine peptone. The $P$. Ostreatus strain was incubated at $25^{\circ} \mathrm{C}$ and the $F$. fomentarius-EUM1 strain at $36^{\circ} \mathrm{C}$. The dry mass of the biomass that grew after 7 days of incubation in mediums M1 and M2 were separated from the agar boiling it in distilled water and drying it for 24 hours at $60^{\circ} \mathrm{C}$. The effect of mycelial growth on the culture methods evaluated (M1 and M2) was analyzed in a completely randomized experimental design with a $2^{2}$ factorial arrangement. Data was analyzed with the GLM procedure of $S{ }^{\circledR}$ and means were compared with Tukey test $(p \leq 0.05)$ to differentiate groups.

Solid medium fermentation. SF with each strain was done by placing $40 \mathrm{~g}$ of SCB $(0.5 \mathrm{~cm} \varnothing$ and $85 \%$ moisture) in an Erlenmeyer flask. It was then

\section{MATERIALES Y MÉTODOS}

Sitio de estudio. El trabajo fue realizado en los laboratorios de Microbiología del Departamento de Zootecnia de la Universidad Autónoma Chapingo, y en el Colegio de Postgraduados, ambos del Estado de México.

Inóculos. Se utilizaron dos cepas de los hongos Pleurotus ostreatus-IE8 y Fomes fomentariusEUM1, ambas con potencial para producir enzimas lignocelulósicas. La cepa EUM1 fue obtenida de la colección de cultivos de la Universidad Autónoma Metropolitana y la cepa IE8 del Colegio de postgraduados.

Medio de cultivo: Las cepas fueron reactivadas inicialmente en agar-extracto de malta y se propagaron en un sustrato de inicio empleando una mezcla de grano de sorgo-BCA en una relación 80:20, para su posterior uso en la FS con BCA.

Experimentos. Se realizaron dos experimentos: en el primero se evaluó la actividad enzimática, velocidad de crecimiento radial (Vcr) y biomasa de las cepas de los hongos $P$. ostreatus-IE8 y $F$. fomentarius-EUM1. En el segundo se determinó la cinética de fermentación in vitro por la técnica de producción de gas (6) del BCA tratado 7 d por FS con las cepas de hongos $P$. ostreatus-IE8, evaluación del ECE de 7 d con $P$. ostreatus-IE8, y fueron comparados con una enzima comercial (Fibrozyme ${ }^{\circledR}$, Alltech Inc., Nicholasville, KY) y un testigo, además de evaluar la composición química de los tratamientos, control y testigo de estudio.

Determinación de la velocidad de crecimiento radial (Vcr) y de la MS de la biomasa. La Vcr para ambas cepas se midió durante $7 \mathrm{~d}$ en dos medios de cultivo usando el método de Trinci (7): Medio 1 (M1) con extracto de bagazo de caña de azúcar (EB) obtenido al hervir $100 \mathrm{~g}$ de BCA en $1 \mathrm{~L}$ de agua destilada por 30 min más agar-agar $(1.5 \%)$ y peptona de caseína $(0.5 \%)$; y Medio 2 (M2) con EB y agar-agar (1.5\%) sin peptona de caseína. La cepa $P$. ostreatus se incubó a $25^{\circ} \mathrm{C}$ y $36^{\circ} \mathrm{C}$ para $F$. fomentarius-EUM1. La MS de la biomasa que creció a los 7 d de incubación en los medios M1 y M2 se separó del agar hirviéndolos en agua destilada y secando por $24 \mathrm{~h}$ a $60^{\circ} \mathrm{C}$. El efecto del crecimiento micelial en los medios de cultivo evaluados (M1 y M2) se analizó en un diseño experimental completamente al azar con un arreglo factorial $2^{2}$. Los datos se analizaron con el procedimiento GLM de $\operatorname{SAS}^{\circledR}(8)$ y las medias se compararon con la prueba de Tukey $(p \leq 0.05)$ para diferenciar entre grupos. 
sterilized for $15 \mathrm{~min}$ at $15 \mathrm{PSI}$ and $121^{\circ} \mathrm{C}$. The inoculation was done with 10 agar-malt extract cylinders with mycelium $(1 \mathrm{~cm} \varnothing)$ of $P$. ostreatusIE8 incubated at $25^{\circ} \mathrm{C}$ and $36^{\circ} \mathrm{C}$ for $F$. fomentariusEUM1. In both cases the SF was evaluated at 0.7 and $15 \mathrm{~d}$.

Determining enzymatic activity. In both strains the crude enzymatic extracts (CEE) obtained from SF at 0.7 and 15 days of fermentation were evaluated (four independent samples were gathered per day). The CEE was obtained by macerating the solid ferment of each strain for 20 min in distilled water at a ratio of $1: 1.5$. This was filtered and pressed by centrifugation for $20 \mathrm{~min}$ at 12.000 rpm at $4^{\circ} \mathrm{C}$. Xylanase and cellulose activities were determined with the Miller method (9), laccase with ABTS according to Klis et al (10) and extracellular protein following the Bradford method (11). The experimental design used separate areas, divided in time, and was distributed randomly. Data was analyzed with the $\mathrm{SAS}^{\circledR}$ GLM procedure (8), and means was compared with the Tukey test ( $p \leq 0.05)$ to differentiate days of FS and experimental strains. The extracellular protein and laccase variables were transformed at logarithmic scale $(y+1)$ to ensure data normality.

Enzymatic activity was expressed in international units (IU) per gram of initial dry substrate (IDS) in which one IU was defined as the quantity of enzymes released by $1 \mu \mathrm{mol}$ xylose or glucose per min under the reaction conditions indicated.

Evaluation of in vitro kinetic fermentation of SCB. Four treatments were evaluated, one control, each in a Erlenmeyer flask with $100 \mathrm{~g} \mathrm{SCB}$ as substrate $(0.5 \mathrm{~cm} \varnothing$ and $85 \%$ moisture), sterilized for $20 \mathrm{~min}$ at $15 \mathrm{PSI}$ and $121^{\circ} \mathrm{C}$. A determined amount of freshly collected microbial inoculum, SCB fermented for $7 \mathrm{~d}$, CEE from the $7^{\text {th }}$ day SF, commercial cellulase (Fibrozyme ${ }^{\circledR}$ ) as control and a control without enzymes. Treatments were classified as: FSi: $5 \mathrm{~g}$ of freshly collected inoculum from strain $P$. ostreatus-IE8; FSc: $5 \mathrm{~g}$ of SF (7d) in SCB with strain P.ostreatus-IE8; FS-ECE: $5 \mathrm{~g}$ of freshly collected inoculum from strain $P$. ostreatusIE8 and $1.9 \mathrm{~mL}$ of CEE from SF (7d) from the same strain. CEE: $1.9 \mathrm{~mL}$ of CEE from FS (7d) of strain $P$. ostreatus-IE8; Control: $0.2 \mathrm{~mL}$ of commercial enzyme (Fibrozyme ${ }^{\circledR}$ ); control: SCB without inoculum. The inoculum $P$. ostreatus-IE8 was obtained by spreading the strain on sorghum grainSCB substrate in a proportion of $80: 20$, respectively. The SF and CEE were obtained at $7 \mathrm{~d}$ fermentation with $P$. ostreatus-IE8. The 7d Solid Fermented CEE and the commercial xylanase both showed the same results of 8.5 IU of xylanase; corresponding to diluting $1 \mathrm{~g}$ of commercial xylanase in $10 \mathrm{~mL}$ of distilled water ( $\left.1 \mathrm{~g} \mathrm{~kg}^{-1} \mathrm{MS}\right)$. The CEE treatments
Fermentación en medio sólido. La FS con cada cepa se realizó colocando $40 \mathrm{~g}$ de BCA $(0.5 \mathrm{~cm}$ $\varnothing$ y $85 \%$ de humedad) en un matraz Erlenmeyer y se esterilizó por $15 \mathrm{~min}$ a 15 PSI y $121^{\circ} \mathrm{C}$. La inoculación se realizó con 10 cilindros de agarextracto de malta con micelio $(1 \mathrm{~cm} \varnothing)$ de $P$. ostreatus-IE8 que se incubó a $25^{\circ} \mathrm{C}$ y $36^{\circ} \mathrm{C}$ para F. fomentarius-EUM1; en ambos casos la FS se evaluó durante 0,7 y $15 \mathrm{~d}$.

Determinación de actividad enzimática. Para ambas cepas se evaluaron los extractos crudos enzimáticos (ECE) obtenidos de la FS a 0,7 y 15 d de fermentación (se obtuvieron cuatro muestras independientes por tiempo). Los ECE se obtuvieron macerando el fermentado sólido de cada cepa durante 20 min con agua destilada en una relación $1: 1.5$, se filtraron y se prensaron para posteriormente centrifugar por $20 \mathrm{~min}$ a $12.000 \mathrm{rpm}$ y $4^{\circ} \mathrm{C}$. La actividad de xilanasas y celulasas se determinó por el método de Miller (9), lacasas con ABTS según Klis et al (10) y proteína extracelular según Bradford (11). El diseño experimental fue de parcelas divididas en el tiempo distribuido completamente al azar. Los datos se analizaron con el procedimiento GLM de $\operatorname{SAS}^{\circledR}(8)$ y las medias se compararon con la prueba de Tukey $(p \leq 0.05)$ para diferenciar entre días de FS y cepas experimentales. Las variables proteína extracelular y lacasas se transformaron a escala logarítmica $(y+1)$ para cumplir con la normalidad de los datos.

La actividad enzimática se expresó en unidades internacionales (UI) por gramo de sustrato seco inicial (SSi), donde una UI se definió como la cantidad de enzima que libera $1 \mu \mathrm{mol}$ de xilosa o glucosa por min en las condiciones de reacción indicadas.

Evaluación de la cinética de fermentación in vitro del BCA. Se evaluaron 4 tratamientos, un control y un testigo cada uno en matraces Erlenmeyer con $100 \mathrm{~g}$ BCA como sustrato (0.5 $\mathrm{cm} \varnothing$ y $85 \%$ de humedad) esterilizados $20 \mathrm{~min}$ a 15 PSI y $121^{\circ} \mathrm{C}$; posteriormente a estos se les añadió una cantidad conocida de inóculo microbiano fresco, BCA fermentado durante 7d, ECE del día siete de FS, celulasa comercial (Fibrozyme ${ }^{\circledR}$ ) como testigo y un control sin enzima. Los tratamientos se clasificaron como: FSi: $5 \mathrm{~g}$ de inóculo fresco de la cepa $P$. ostreatusIE8; FSc: $5 \mathrm{~g}$ de FS (7d) en BCA con cepa P.ostreatus-IE8; FS-ECE: $5 \mathrm{~g}$ de inóculo fresco de la cepa $P$. ostreatus-IE8 y $1.9 \mathrm{~mL}$ de ECE del FS (7d) de la misma cepa; ECE: $1.9 \mathrm{~mL}$ de ECE del FS (7d) de la cepa $P$. ostreatus-IE8; Testigo: 0.2 $\mathrm{mL}$ de enzima comercial (Fibrozyme ${ }^{\circledR}$ ); Control: BCA sin inóculo. El inóculo de $P$. ostreatusIE8 se obtuvo al propagar la cepa en sustrato 
(1.9 MI) had: $1.7 \mathrm{IU}$ of xylanase and 0.01 cellulase. All samples were fermented for $7 \mathrm{~d}$ at $25^{\circ} \mathrm{C}$, they were dried for $96 \mathrm{~h}$ at $60^{\circ} \mathrm{C}$ for nutrient analysis. An analysis was made on: dried matter (DM), ash, organic matter (OM), crude protein (CP), hemicellulose, cellulose, lignin acid detergent (LAD) according to AOAC (12), neutral detergent fiber (NDF) and acid detergent fiber (ADF) according to Van Soest, et al (13).

The kinetics of gas production was done with the Menke and Steigass method (6). A $0.5 \mathrm{~g}$ sample of a treatment and $90 \mathrm{~mL}$ of standardized ruminant inoculum were placed in ambar glasses with a flow of $\mathrm{CO}_{2}$ The glasses were placed on a double boiler at $39^{\circ} \mathrm{C}$. Gas pressure was measured at $0,2,4,10$, $14,18,24,30,36,48,60$ and $72 \mathrm{~h}$ of incubation. The transformation from pressure to gas volume was adjusted with a lineal regression model. The gas production scale $(\mathrm{S})$, maximum volume of produced gas $(\mathrm{Vm})$ and lag time $(\mathrm{L})$ were obtained through the logistic model $\mathrm{Va}=\mathrm{Vm} /(1+\exp (2-4 * S *(T-L)))(14)$. The final residues were filtered in ANKOM bags and dried for $24 \mathrm{~h}$ at $60^{\circ} \mathrm{C}$. DM degradation (DEGMS) and real degradation (DEGV) (15) were calculated.

The experimental design was a random element. Data was analyzed using the GLM procedure of SAS $^{\circledR}(8)$. Means were compared with the Tukey test $(p \leq 0.05)$. Correlation was calculated using the CORR (8) procedure. Variables L, DEGMS and DEGV were made into square root, while \%OM, $\% A F D$ and $\%$ hemicellulose were transformed using a logarithmic scale $(y+1)$ to fulfill data normality requirements.

\section{RESULTS}

The RGR of $P$. ostreatus-IE8 in culture medium without peptone (M2) was superior $(p \leq 0.05)$ to culture medium with peptone (M1), while fomentarius-EUM1did not show any difference between the culture mediums evaluated $(p>0.05)$. Strains comparison did not show significant differences either $(p>0.05)$. The biomass content was higher for $F$. fomentarius-EUM1 $(p \leq 0.05)$ in both culture mediums (Table 1 ).

Table 1. Radial growth velocity (Rgv) and biomass produced by $P$.ostreatus-IE 8 and $F$. fomentarius-EUM1 fungi in culture medium with and without casein peptone $(0.5 \%)$.

\begin{tabular}{lccc}
\hline \multicolumn{1}{c}{ Strain } & Medium & $\mathbf{R g v}\left(\mathbf{m m ~ h} \mathbf{h}^{-\mathbf{1}}\right)$ & Biomass (mg) \\
\hline \multirow{2}{*}{ F. Fomentarius-EUM1 } & M1 & $0.618^{\mathrm{a}}$ & $53.0^{\mathrm{a}}$ \\
& M2 & $0.613^{\mathrm{a}}$ & $48.0^{\mathrm{ab}}$ \\
P. Ostreatus IE8 & M1 & $0.406^{\mathrm{b}}$ & $33.8^{\mathrm{b}}$ \\
& M2 & $0.616^{\mathrm{a}}$ & $37.5^{\mathrm{ab}}$ \\
\hline
\end{tabular}

Means in row with different text are different $(p \leq 0.05)$

${ }^{*}$ Formulated with sugarcane bagasse and agar-agar (1.5\%); M1, with caseine peptone $(0.5 \%)$ as nitrogen source; M2 without caseine peptone. grano de sorgo-BCA en una proporción 80:20, respectivamente. EI FS y ECE se obtuvieron de 7 $\mathrm{d}$ de fermentación con $P$. ostreatus-IE8. EI ECE de los $7 \mathrm{~d}$ de FS y xilanasa comercial se igualaron a 8.5 UI de xilanasas, correspondientes a la dilución de $1 \mathrm{~g}$ xilanasa comercial en $10 \mathrm{~mL}$ de agua destilada ( $\left.1 \mathrm{~g} \mathrm{~kg}^{-1} \mathrm{MS}\right)$. Los tratamientos con ECE (1.9 mL) contenía: 1.7 UI de xilanasas, $0.1 \mathrm{UI}$ de celulasas y $6.4 \mathrm{UI}$ de lacasas, mientras la enzima comercial $(0.2 \mathrm{~mL})$ contenía: $1.7 \mathrm{UI}$ de xilanasas y 0.01 UI de celulasas. Todas las muestras se fermentaron $7 \mathrm{~d}$ a $25^{\circ} \mathrm{C}$, se secaron $96 \mathrm{~h}$ a $60^{\circ} \mathrm{C}$ para el análisis nutrimental. Se realizó un análisis de materia seca (MS), cenizas, materia orgánica (MO), proteína cruda $(P C)$, hemicelulosa, celulosa, lignina detergente ácido (LDA) según AOAC (12), fibra detergente neutro (FDN) y fibra detergente ácido (FDA) según Van Soest, et al (13).

La cinética de producción de gas se realizó por el método de Menke y Steigass (6). En frascos de vidrio color ámbar, con flujo de $\mathrm{CO}_{2}$ se colocaron $0.5 \mathrm{~g}$ de muestra de un tratamiento y $90 \mathrm{~mL}$ de inóculo ruminal estandarizado. Los frascos se incubaron en baño maría a $39^{\circ} \mathrm{C}$. La presión de gas se midió a $0,2,4,10,14,18,24,30,36$, 48,60 y 72 h de incubación. La transformación de presión a volumen de gas se ajustó con un modelo de regresión lineal. La tasa de producción de gas (S), volumen máximo de gas producido $(\mathrm{Vm})$ y tiempo Lag $(L)$ se obtuvieron con el modelo logístico $\mathrm{Va}=\mathrm{Vm} /(1+\exp (2-4 * S *(T-L)))(14)$. El residuo final se filtró en bolsas ANKOM y se secaron $24 \mathrm{~h}$ a $60^{\circ} \mathrm{C}$. Se calculó la degradación de la MS (DEGMS) y degradación verdadera (DEGV; 15).

El diseño experimental fue un completamente al azar, los datos se analizaron con el procedimiento GLM de SAS $^{\circledR}(8)$. Las medias se compararon con la prueba de Tukey $(p \leq 0.05)$. La correlación se calculó con el procedimiento CORR (8). Las variables $L$, DEGMS y DEGV se transformaron a raíz cuadrada, mientras \%MO, \%FDA y $\%$ Hemicelulosa se transformaron a una escala logarítmica $(\mathrm{y}+1)$ para cumplir con la normalidad de los datos.

\section{RESULTADOS}

La Vor de $P$. ostreatus-IE8 en medio de cultivo sin peptona (M2) fue superior $(p \leq 0.05)$ al medio de cultivo con peptona (M1), mientras que $F$. fomentarius-EUM1 no mostró diferencias entre medios de cultivo evaluados $(p>0.05)$, y la comparación entre cepas tampoco mostraron diferencias significativas $(p>0.05)$. El contenido de biomasa fue mayor para $F$. fomentarius-EUM1 $(p \leq 0.05)$ en ambos medios de cultivo (Tabla 1). 
Table 2 shows that both fungi strains at $7 d$ had increased enzyme activity, xylanase concentration did not vary $(p>0.05)$ between strains on the $7 \mathrm{~d}$ of SF. The $P$. ostreatus-IE8 strain had a greater laccase activity (10.65 UI $\mathrm{g}^{-1} \mathrm{SSi}$ ) at $7 \mathrm{~d}$, while $F$. fomentarius-EUM1 had greater cellulase activity $\left(1.90 \mathrm{UI} \mathrm{g}^{-1} \mathrm{SSi}\right)$ at $7 \mathrm{~d}$ of SF. The $P$. ostreatus-IE8 strain produced a higher concentration of extracellular protein coinciding with the $7 \mathrm{~d}$ of SF $\left(0.52 \mathrm{mg} \mathrm{g}^{-1} \mathrm{SSi}\right.$; Table 2$)$.

Table 2. Enzymatic activities and extracellular protein concentration of crude enzyme extract of $P$. ostreatus-IE8 and $F$. fomentarius-EUM1 at different solid fermentation days (SF).

\begin{tabular}{|c|c|c|c|c|c|}
\hline \multirow{2}{*}{ Strain } & \multirow{2}{*}{$\begin{array}{c}\text { Day } \\
\text { SF }\end{array}$} & \multirow{2}{*}{$\begin{array}{c}\text { Xylanase } \\
\text { (UI g g-1 }^{\text {SSi) }}\end{array}$} & \multirow{2}{*}{$\begin{array}{c}\text { Laccase } \\
\begin{array}{c}\text { (UI g } \mathbf{g}^{-1} \\
\text { SSi) }\end{array}\end{array}$} & \multirow{2}{*}{$\begin{array}{c}\text { Cellulase } \\
\begin{array}{c}\text { (UI g-1 } \\
\text { SSi) }\end{array}\end{array}$} & \multirow{2}{*}{$\begin{array}{c}\begin{array}{c}\text { Extracellular } \\
\text { protein }\end{array} \\
\left(\mathrm{mg} \mathrm{g}^{-1} \mathrm{SSi}\right) \\
\end{array}$} \\
\hline & & & & & \\
\hline \multirow{3}{*}{$\begin{array}{c}\text { F. Fomentarius- } \\
\text { EUM1 }\end{array}$} & 0 & $4.24^{\mathrm{cd}}$ & $0.70^{\mathrm{cd}}$ & $0.43^{c}$ & $0.18^{\text {cd }}$ \\
\hline & 7 & $5.50 \mathrm{ab}$ & $0.96^{c}$ & $1.90^{\mathrm{a}}$ & $0.39^{b}$ \\
\hline & 15 & $4.85 b c$ & $4.52^{b}$ & $1.33^{\mathrm{b}}$ & $0.38^{b}$ \\
\hline \multirow{3}{*}{ P. Ostreatus IE8 } & 0 & $4.05^{\mathrm{cd}}$ & $0.00^{d}$ & $0.39^{c}$ & $0.23^{c}$ \\
\hline & 7 & 6.32 a & $10.65^{a}$ & $0.55^{c}$ & $0.52^{a}$ \\
\hline & 15 & $3.53^{d}$ & $0.17^{c d}$ & $0.30^{c}$ & $0.11^{d}$ \\
\hline
\end{tabular}

Means in row with different letter are different $(p \leq 0.05)$.

Table 3 shows the results of the evolution of maximum gas production volume $(\mathrm{Vm})$, dried matter degradation (DEGMS), and real degradation (DEGV). FSi, FSC and SF-CEE methods had the lowest Vm, DEGM and DEGV values, with no difference among them ( $p>0.05)$. CEE treatments and control methods produced values higher than $100 \mathrm{~mL} \mathrm{~g} \mathrm{MS}^{-1}$, with control showing the highest Vm (123.80) $\mathrm{mL} \mathrm{g} \mathrm{MS}^{-1}$ ). The CEE and control method did not increase DEGMS and DEGV when compared to the control method.

The fractions of NDF, ADF and ash from all methods decreased when compared to the control method $(p \leq 0.05)$. In contrast, the fractions of $L A D, C P$ and OM increased in comparison to the control method. The methods with added SF of $7 \mathrm{~d}$ had higher values of $L A D, C P$ and ash $(p \leq 0.05)$ in relation to those with added enzymes (CEE and control) (Table 4).

Table 3. Variables of in vitro gas production from sugarcane bagasse treated with commercial enzymes, solid fermentation or crude enzymatic extract of $P$. ostreatus-IE8.

\begin{tabular}{|c|c|c|c|c|c|c|}
\hline Variable & $\mathbf{F S i}$ & FSc & FS-ECE & ECE & Witness & Control \\
\hline $\mathrm{Vm}\left(\mathrm{mL} g \mathrm{MS}^{-1}\right)$ & $47.57^{e}$ & $51.44^{\mathrm{de}}$ & $53.03^{d}$ & $113.60^{\mathrm{b}}$ & $104.03^{c}$ & $123.80^{\mathrm{a}}$ \\
\hline$S\left(h^{-1}\right)$ & $0.019^{c}$ & $0.021^{b c}$ & $0.021^{b}$ & $0.028^{a}$ & $0.027^{a}$ & $0.028^{a}$ \\
\hline$L(h)$ & $12.22^{\mathrm{b}}$ & $17.29^{a}$ & $05.37^{c}$ & $12.88^{b}$ & $13.98^{\mathrm{ab}}$ & $10.82^{\mathrm{b}}$ \\
\hline DEGMS (\%) & $19.85^{c}$ & $20.33^{c}$ & $20.84^{c}$ & $39.25^{\mathrm{ab}}$ & $36.25^{\mathrm{b}}$ & $42.68^{a}$ \\
\hline DEGV (\%) & $27.41^{c}$ & $28.67^{c}$ & $27.03^{c}$ & $44.59^{b}$ & $44.23^{b}$ & $50.38^{a}$ \\
\hline
\end{tabular}

Means in row with different letter are different $(p \leq 0.05)$.

FSi: $5 \mathrm{~g}$ of de $P$. ostreatus-IE8; FSc inoculum: $5 \mathrm{~g}$ of solid fermentation of SCB with strain $P$. ostreatus-IE8 (7d); SF-CEE: $5 \mathrm{~g}$ of $P$. ostreatus-IE8 inoculum and CEE from the same strain fermented for 7d; CEE: $1.9 \mathrm{~mL}$ of $P$. ostreatus-IE8 CEE fermented for $7 \mathrm{~d}$; Control: $0.2 \mathrm{~mL}$ of commercial enzyme (Fibrozyme ${ }^{\circledR}$ ); Control: BCA without inoculum .
En la tabla 2, se muestra que ambas cepas de hongos a los 7d presentaron la mayor actividad de enzimas. La concentración de xilanasas no mostró diferencias $(p>0.05)$ entre cepas en el 7d de FS. La cepa $P$. ostreatus-IE8 mostró mayor actividad de lacasas (10.65 $\mathrm{UI} \mathrm{g}^{-1} \mathrm{SSi}$ ) al 7d, mientras que $F$. fomentarius-EUM1 mostró mayor actividad de celulasas (1.90 UI $\left.\mathrm{g}^{-1} \mathrm{SSi}\right)$ para 7d de FS. La cepa P. ostreatusIE8 produjo la mayor concentración de proteína extracelular coincidiendo el $7 \mathrm{~d}$ de FS $(0.52 \mathrm{mg}$ $\mathrm{g}^{-1} \mathrm{SSi}$; Tabla 2).

En la tabla 3, se muestran los resultados de la evolución del volumen máximo de producción de gas $(V m)$, degradación de la materia seca (DEGMS) y degradación verdadera (DEGV). Los tratamientos FSi, FSc y FS-ECE mostraron los valores más bajos de Vm, DEGMS y DEGV, sin diferencias entre ellos $(p>0.05)$. Los tratamientos ECE, testigo y control produjeron valores mayores a $100 \mathrm{~mL} \mathrm{~g} \mathrm{MS}^{-1}$, donde control mostró el mayor $\mathrm{Vm}\left(123.80 \mathrm{~mL} \mathrm{~g} \mathrm{MS}^{-}\right.$ ${ }^{1}$ ). El tratamiento ECE y el testigo no mejoraron DEGMS y DEGV comparados con el control.

La fracción de FDN, FDA y cenizas de todos los tratamientos disminuyeron respecto al control $(p \leq 0.05)$; en contraste, aumentaron la fracción de LDA, PC y MO en comparación al control. Los tratamientos donde se adicionó FS de 7d mostraron valores altos de LDA, PC y cenizas $(p \leq 0.05)$ en relación aquellos donde se adicionaron enzimas (ECE y testigo) (Tabla 4).

\section{DISCUSIÓN}

Los hongos basidiomicetos $P$. ostreatus y $F$. fomentarius tienen la capacidad de producir simultáneamente enzimas hidrolíticas y

Table 4. Nutrient composition of sugarcane bagasse treated with commercial enzyme, solid fermentation and crude enzymatic extract of $P$. ostreatus-IE8 fungi.

\begin{tabular}{lcccccc}
\hline \multicolumn{1}{c}{ Variable } & FSi & FSc & SF-CEE & CEE & Witness Control \\
\hline FND (\%) & $69.25^{\mathrm{d}}$ & $71.68^{\mathrm{bcd}}$ & $70.25^{\mathrm{cd}}$ & $74.53^{\mathrm{b}}$ & $73.48^{\mathrm{bc}}$ & $81.20^{\mathrm{a}}$ \\
ADF (\%) & $42.75^{\mathrm{ab}}$ & $44.00^{\mathrm{ab}}$ & $41.08^{\mathrm{bc}}$ & $41.65^{\mathrm{abc}}$ & $44.78^{\mathrm{a}}$ & $52.17^{\mathrm{c}}$ \\
LAD (\%) & $19.38^{\mathrm{a}}$ & $20.08^{\mathrm{a}}$ & $17.23^{\mathrm{b}}$ & $15.68^{\mathrm{c}}$ & $16.25^{\mathrm{bc}}$ & $11.63^{\mathrm{d}}$ \\
$\begin{array}{l}\text { Hemicellulose } \\
\text { (\%) }\end{array}$ & $26.50^{\mathrm{c}}$ & $27.68^{\mathrm{bc}}$ & $29.20^{\mathrm{b}}$ & $31.40^{\mathrm{a}}$ & $28.73^{\mathrm{bc}}$ & $29.03^{\mathrm{b}}$ \\
Cellulose (\%) & $23.38^{\mathrm{c}}$ & $23.93^{\mathrm{c}}$ & $23.88^{\mathrm{c}}$ & $25.95^{\mathrm{c}}$ & $28.55^{\mathrm{b}}$ & $40.53^{\mathrm{a}}$ \\
CP (\%) & $4.28^{\mathrm{a}}$ & $3.18^{\mathrm{b}}$ & $4.48^{\mathrm{a}}$ & $2.68^{\mathrm{c}}$ & $2.64^{\mathrm{c}}$ & $1.63^{\mathrm{d}}$ \\
Ash (\%) & $14.25^{\mathrm{a}}$ & $14.46^{\mathrm{a}}$ & $14.05^{\mathrm{a}}$ & $14.35^{\mathrm{a}}$ & $13.58^{\mathrm{a}}$ & $5.30^{\mathrm{b}}$ \\
OM (\%) & $85.75^{\mathrm{a}}$ & $85.54^{\mathrm{a}}$ & $85.95^{\mathrm{a}}$ & $85.65^{\mathrm{a}}$ & $86.42^{\mathrm{a}}$ & $94.70^{\mathrm{a}}$ \\
\hline
\end{tabular}

Means in row with different letter are different ( $\leq \leq 0.05)$.

FSi: $5 \mathrm{~g}$ of $P$. ostreatus-IE8 inoculum; FSc: $5 \mathrm{~g}$ of solid fermented SCB with $P$. ostreatus-IE8 strain (7d); SF-CEE: $5 \mathrm{~g}$ of $P$. ostreatus-IE8 inoculum and CEE from the same strain fermented for 7d; CEE: $1.9 \mathrm{~mL}$ of $7 \mathrm{~d}$ fermented CEE from $P$. ostreatus-IE8; Control: $0.2 \mathrm{~mL}$ of commercial enzyme (Fibrozyme ${ }^{\natural}$ ); Control: SCB without inoculum. 


\section{DISCUSION}

Basidiomycete fungi $P$. ostreatus and $F$. fomentarius have the capacity to simultaneously produce hydrolytic and lignolitic enzymes by SF (16). Marquez et al (17) conducted an FS test for $14 \mathrm{~d}$ at an incubation temperature of $34^{\circ} \mathrm{C}$ with F. fomentarius-EUM1 on SCB and $80 \%$ moisture. Xilanase (147.27 UI g $\left.{ }^{-1} \mathrm{SSi}\right)$ and cellulase (8.51 UI $\mathrm{g}^{-1} \mathrm{SSi}$ ) activity was reported, but lower on laccase (3.45 UI g-1 SSi). Membrillo et al (18) used $P$. ostreatus-IE 8 in a SF for $8 \mathrm{~d}$ on SCB with a particle size of $2.9 \mathrm{~mm}$ and $80 \%$ moisture. The xylanase activity was reduced (5.79 $\mathrm{U} \mathrm{g}^{-1} \mathrm{SSi}$ ) as well as laccase $\left(0.021 \mathrm{U} \mathrm{g}^{-1} \mathrm{SSi}\right)$.

The SF process has important considerations: particle size, type of substrate, microorganisms, nature of inoculum, temperature and duration $(3,4)$. For those reasons, and based on the results of this research, it is important to consider the enzyme-substrate affinity for this type of biotechnological developments because of the great variations that exist. The chemical characteristics of SCB make it possible for the $P$. ostreatus-IE8 strain to have great potential to produce laccase enzymes and act specifically on the lignin, while $F$. fometarius has a greater cellulase production capacity, acting directly on the cellulose on the cell wall of fibrous forages used to feed ruminants (1)

It is unknown if adding complex organic nitrogen sources like casein peptone can have an inhibiting effect on radial growth speed and biomass production, given that existing literature only includes mineral nitrogen. Membrillo et al (18) used urea and ammonia sulfate as a source of organic nitrogen in a malt extract medium to grow $P$. ostreatus-IE8, resulting in inferior rsg $\left(0.234\right.$ y $\left.0.308 \mathrm{~mm} \mathrm{~h}^{-1}\right)$. Levin et al (19) tried using different nitrogen sources for the production of laccase by three basidiomycetes fungi, without finding any differences in the laccase production patterns given the medium composition. Therefore, the addition of nitrogenized sources to culture mediums for the growth of basidiomycete fungi does not increase the fungal rsg or enzyme production $(18,19)$.

The kinetics of in vitro gas production results from the anaerobic fermentation of soluble carbohydrates or cellular wall with microorganisms from the rumen. The FSi, FS-CEE treatments presented less values in the production kinetics of in vitro gas (Table 3 ) because they result from a fermentation process where $P$. ostreatus-IE8 consumes soluble carbohydrates and hemicellulose as primary lignolíticas por FS (16). Márquez et al (17) realizaron una $\mathrm{FS}$ durante $14 \mathrm{~d}$ a $34^{\circ} \mathrm{C}$ de incubación con $F$. fomentarius-EUM1 sobre BCA con $80 \%$ de humedad, reportando una actividad de xilanasas (147.27 UI g ${ }^{-1} \mathrm{SSi}$ ) y celulasas (8.51 $\left.\mathrm{UI} \mathrm{g}^{-1} \mathrm{SSi}\right)$, pero menor en lacasas (3.45 UI g ${ }^{-1} \mathrm{SSi}$ ). Membrillo et al (18) usaron $P$. ostreatus-IE8 en una FS de $8 \mathrm{~d}$ sobre BCA con un tamaño de partícula de $2.9 \mathrm{~mm}$ y $80 \%$ de humedad, donde obtuvieron menor actividad de xilanasas $\left(5.79 \mathrm{U} \mathrm{g}^{-1} \mathrm{SSi}\right)$ y lacasas (0.021 U g ${ }^{-1}$ SSi).

El proceso de FS tiene importantes consideraciones que involucran tamaño de partícula, tipo de sustrato, microorganismo, naturaleza del inóculo, temperatura y duración de la propia FS $(3,4)$. Por lo anterior, y con base en los resultados de esta investigación es importante considerar la afinidad enzimasustrato para este tipo de desarrollos biotecnológicos, ya que existe gran variación en los mismos. Las características químicas del bagazo de caña de azúcar permiten a la cepa del hongo $P$. ostreatus-IE8 tener un alto potencial para producir enzimas lacasas y actuar específicamente sobre la lignina, mientras que $F$. fometarius tiene mayor producción de celulasas que actúa directamente sobre la celulosa de la pared celular de forrajes altos en fibra usados comúnmente en la alimentación de rumiantes (1).

Se desconoce si la adición de fuentes complejas de nitrógeno orgánico como la peptona de caseína puede tener un efecto inhibitorio en la velocidad de crecimiento radial y producción de biomasa, ya que la literatura sólo indica el uso de nitrógeno mineral. Membrillo et al (18) utilizaron urea y sulfato de amonio como fuente de nitrógeno inorgánico en medios de extracto de malta para el crecimiento de $P$. ostreatus-IE8, obteniendo valores inferiores de $\operatorname{Vcr}\left(0.234\right.$ y $\left.0.308 \mathrm{~mm} \mathrm{~h}^{-1}\right)$. Levin et al (19) probaron el uso de diferentes fuentes de nitrógeno en la producción de lacasas por tres hongos basidiomicetos sin hallar diferencias en los patrones de producción de lacasas por la composición del medio. Por lo anterior, la adición de fuentes nitrogenadas en medios de cultivo para el crecimiento de hongos basidiomicetos, no incrementa la Vcr fúngica o producción enzimática $(18,19)$.

La cinética de producción de gas in vitro se obtiene de una fermentación anaerobia de los carbohidratos solubles o pared celular con microorganismos del rumen. Los tratamientos FSi, FSC y FS-ECE presentaron menores valores en la cinética de producción de gas 
sources of energy for growth $(2,20)$. CEE and the control treatment had a larger amount of carbohydrates available because they were not predigested. The CEE and control treatment showed different behaviors in the gas production kinetics when compared with the control treatment, even when the enzymes produced by SF promotes greater availability of structural and soluble carbohydrates on the cell wall as a result of the degradation of the substrate, producing more gas in in vitro studies (20).

The increase of LAD and ash fraction, as well as the decrease of the NDF, ADF, cellulose and OM fraction in the $\mathrm{FSi}, \mathrm{FSC}$ and SF-CEE treatments are attributed to the fact that the $P$. ostreatus-IE8 fungi consumes a large amount of carbohydrates, (2) necessary for its development. The increase of CP in the FSi, FSc and SF-CEE treatments is the result of the increase of microbial protein produced directly in the development of the $P$. ostreatus-IE8 fungi on SCB in the SF process (21). The relation between chemical composition and variables in the kinetics of in vitro gas production are used to estimate the digestibility of a substrate by a ruminant (22). The $\mathrm{Vm}$ of gas was positively correlated with NDF, hemicellulose and cellulose, but negatively with LAD (Table 5). Andres et al (23) reported similar findings. His correlations indicate the same tendencies as this study. The negative correlation of $\mathrm{CP}$ with the kinetic variable of gas production is explained because the protein is used in the biosynthesis of microbial protein, and in case microorganisms increase their immediate demand for energy, it catabolizes into an energy source. The positive correlation between DEGMS and DEGV with NDF, cellulose and hemicellulose, but negative with $L A D$ and $C P$ are due to the double fermentation process (solid and anaerobic) that the SCB underwent. Bindelle et al (22) reported negative correlations with NDF and positive with CP because they did not apply to the substrates any sort of fermentation previous to the in vitro gas production, allowing for a larger amount of carbohydrates.

Table 5. Correlation coefficient in the chemical bromatologic composition and the in vitro sugarcane bagasse gas production.

\begin{tabular}{lcccccc}
\hline \multicolumn{1}{c}{ Variables } & Vm & S & DEGMS & DEGV & EM & DMO \\
\hline FDN & $0.789^{a}$ & $0.693^{a}$ & $0.717^{a}$ & $0.712^{a}$ & $0.486^{b}$ & $0.507^{a}$ \\
LAD & $-0.867^{a}$ & $-0.837^{a}$ & $-0.850^{a}$ & $-0.809^{a}$ & $-0.967^{a}$ & $-0.957^{a}$ \\
Hemicellulose & $0.654^{a}$ & $0.647^{a}$ & $0.629^{a}$ & $0.610^{a}$ & $0.704^{a}$ & $0.688^{a}$ \\
Cellulose & $0.787^{a}$ & $0.680^{a}$ & $0.753^{a}$ & $0.711^{a}$ & $0.526^{a}$ & $0.568^{a}$ \\
CP & $-0.769^{a}$ & $-0.785^{a}$ & $-0.787^{a}$ & $-0.790^{a}$ & $-0.298^{b}$ & $-0.226^{b}$ \\
\hline${ }^{a}$ Probabilidad significativa $(p \leq 0.05) i^{b}$ no significativa $(p \geq 0.05)$.
\end{tabular}

in vitro (Tabla 3 ) porque provienen de un proceso fermentativo donde $P$. ostreatus-IE8 consume carbohidratos solubles y hemicelulosa como primera fuente de energía para su crecimiento $(2,20)$. El tratamiento ECE, control y testigo dispusieron de mayor cantidad de carbohidratos porque no fueron predigeridos. El tratamiento ECE y testigo mostraron diferente comportamiento en la cinética de producción de gas comparada con el control aun cuando las enzimas producidas por FS promueven mayor disponibilidad de carbohidratos estructurales y solubles de la pared celular derivados de la degradación del sustrato, produciendo mayor producción de gas en estudios in vitro (20)

El incremento de la fracción LDA y cenizas, además de la disminución de la fracción FDN, FDA, celulosa y $\mathrm{MO}$ en los tratamientos FSi, FSc y FS-ECE se atribuyen a que el hongo $P$. ostreatus-IE8 durante la FS consume un alto contenido de carbohidratos (2) para su desarrollo. El incremento de PC en los tratamientos FSi, FSc y FS-ECE es consecuencia del incremento de proteína microbiana que proviene directamente del desarrollo del hongo $P$. ostreatus-IE8 sobre el BCA durante el proceso de FS (21).

La relación entre composición química y variables de la cinética de producción de gas in vitro se emplean para estimar la digestibilidad de un sustrato por el animal rumiante (22). El Vm de gas se correlacionó positivamente con FDN, hemicelulosa y celulosa, pero negativamente con LDA (Tabla $5)$; situación similar a lo reportado por Andrés et al (23) ya que sus correlaciones indican las mismas tendencias que en el presente estudio. La correlación negativa de PC con las variables de la cinética de producción de gas, se explica porque la proteína se usa para biosíntesis de proteína microbiana y en caso que los microorganismos incrementen sus requerimientos inmediatos de energía, se cataboliza como fuente de energía. La correlación es positiva en DEGMS y DEGV con FDN, celulosa y hemicelulosa, pero negativa con LDA y PC se deben al doble proceso de fermentación (sólida y anaerobia) al que fue sometido el bagazo de caña de azúcar. Bindelle et al (22) reportaron correlaciones negativas con FDN y positivas con PC porque no aplicaron a los sustratos ningún tipo de fermentación previa a la producción de gas in vitro lo que propicio una mayor disponibilidad de carbohidratos.

Esta investigación demuestra que el crecimiento micelial de los hongos Pleurotus ostreatus-IE8 
This research shows that the mycelial growth of the Pleurotus ostreatus-IE8 and Fomes fomentarius-EUM1fungi increases when SCB is added to a conventional culture medium. $F$. fomentarius-EUM1 and $P$. ostreatus-IE8 strains have higher enzymatic activity at $7 \mathrm{~d}$ of fermentation on SCB. The crude enzymatic extract ( $7 \mathrm{~d}$ ) of $P$. ostreatus-IE8 on SCB showed similar effects on the kinetics of in vitro gas production ( $72 \mathrm{~h}$ incubation) as the commercial enzymatic product (Fibrozyme ${ }^{\circledR}$ ). The application of lignocellulolytic or a solid fermentation on SCB reduces the production of in vitro gas and the real degradation of substrate. y Fomes fomentarius-EUM1 se incrementa al adicionar extracto de bagazo de caña de azúcar a un medio de cultivo convencional. Las cepas $F$. fomentarius-EUM1 y $P$. ostreatusIE8 tienen mayor actividad enzimática a los 7 d de fermentación sobre BCA. El extracto crudo enzimático ( 7 d) de $P$. ostreatus-IE8 sobre BCA mostró efectos similares en la cinética de producción de gas in vitro ( $72 \mathrm{~h}$ de incubación) al producto enzimático comercial (Fibrozyme ${ }^{\circledR}$ ). La aplicación de enzimas lignocelulolíticas o una fermentación sólida en BCA reduce la producción de gas in vitro y la degradación verdadera del sustrato.

\section{REFERENCES}

1. Sánchez-Santillán P, Meneses MM, TorresSalado N. Production of Lignocellulolytic Enzymes with Pleurotus ostreatus-IE8 by Solid Fermentation and Its Effect on the Chemical Composition of Sugarcane Bagasse. Life Sci J 2015; 12(2s):37-41.

2. Okano K, Fukui S, Kitao R, Usagawa T. Effects of culture length of Pleurotus eryngii grown on sugarcane bagasse on in vitro digestibility and chemical composition. Anim Feed Sci Technol 2007; 136(3-4):240-247.

3. Cuervo L, Folch JL, Quiroz RE. Lignocelulosa como Fuente de azúcares para la producción de etanol. BioTecnología 2009; 13(3):11-25.

4. Kumar SS, Sczakas G, Soccol CR, and Pandey A. Production of Enzymes by Solid-State Fermentation in Current Developments in Solid-State Fermentation. 1a ed. New York: Springer; 2008.

5. Loera CO, Pérez PMCI, Barbosa RJR, and Villaseñor OF. Laccases. In: GuevaraGonzález RG, Torres-Pacheco I, editors. Advances in Agricultural and Food Biotechnololy. Kerela, Indía: Cabi; 2006.

6. Menke $\mathrm{KH}$ and Steingas $\mathrm{H}$. Estimation of the energetic feed value obtained from Chemical analysis and in vitro gas production using rumen fluid. Anim Res Develop 1988; 28(1):7-55.
7. Trinci APJ. A kinetic study of the growth of Aspergillus nidulans and other fungi. J Gen Microbiol 1969; 57(1):11-24.

8. SAS. SAS/STAT Sofware. Version 9.3. Cary, NC SAS, USA: Institute Inc; 2011.

9. Miller GL. Use of dinitrosalicylic acid reagent for determination of reducing sugar. Anal Biochem 1959; 31(3):426-428.

10. Klis M, Rogalski J, Bilewicz R. Voltammetric determination of catalytic reaction parameters of laccase bases on electrooxidation of hydroquinone and ABTS. Bioelectrochemistry 2007; 71(1):2-7.

11. Bradford MM. A rapid and sensitive for the quantitation of microgram quantities of protein-dye binding. Anal Biochem 1976; 72(1-2):248-254.

12. A.O.A.C. Official Methods of Analysis (18th Ed). Washington D.C.: A.O.A.C International; 2005.

13. Van Soest PJ, Robertson JB, Lewis BA. Methods for dietary fiber, neutral detergent fiber, and nonstarch polysaccharides in relation to animal nutrition. J Dairy Sci 1991; 74(10):3583-3597.

14. Schofiel P, and Pell AN. Measurement and kinetic analysis of the neutral detergentsoluble carbohydrate fraction of legumes y grasses. J Anim Sci 1995; 73(11): 3455-3463. 
15. Blümmel $M$, Cone JW, Van Gelber $A H$, Nshalai I, Umunna NN, Makkar HPS, et al. Prediction of forage intake using in vitro gas production methods: Comparison of multiphase fermentation kinetics measured in an automated gas test, and combined gas volume and substrate degradability measurements in a manual syringe system. Anim Feed Sci Technol 2005; $123-$ 124(1):517-526.

16. Elisashvili $\mathrm{V}$, Kachlishvili E and Penninck M. Effect of growth substrate, method of fermentation, and nitrogen source on lignocelluloses-degrading enzymes production by white-rot basidiomycetes. J Ind Microbiol Biotechnol 2008; 35(11):1531-1538.

17. Márquez AAT, Mendoza MGD, González MSS, Buntins DSE y Loera CO. Actividad fibrolítica de enzimas producidas por Trametes $s p$ EUM1, Pleurotus ostreatus IE8 y Aspergillus niger AD96.4 en fermentación sólida. Interciencia 2007; 32(11):780-785.

18. Membrillo I, Sánchez C, Meneses M, Favela E, Loera O. Effect of substrate particle size and additional nitrogen source on production of lignocellulolytic enzymes by Pleurotus ostreatus strains. Bioresource Technol 2008; 99(16):7842-7847.

19. Levin L, Melignani E, Ramos AM. Effect of nitrogen sources and vitamins on ligninolytic enzyme production by some white-rot fungi. Dye decolorization by selected culture filtrates. Bioresource Technol 2010; 101(12):4554-4563.
20. Peláez-Acero A, Meneses-Mayo M, MirandaRomero LA, Ayala-Martínez M, CrosbyGalván MM, Loera-Corral O, y Megías-Rivas MD. Enzimas fibrolíticas producidas por fermentación en estado sólido para mejorar los ensilajes de caña de azúcar. Agrociencia 2011; 45(6):675-685.

21. Akinfemi $A$, Ogunwole OA, Ladipo MK, Adu OA, Osineye OM, and Apata ES. Enhacement of the nutritive value of maize leaf treated with white-rot fungi: Pleurotus sajur caju and Pleurotus pulmonarius, and the effects on chemical composition and in vitro digestibility. Prod Agric Technol 2008; 4(1):106-114.

22. Bindelle J, Ilunga $Y$, Delacollette $M$, MulandKayij M, Umba M, Kindele E and Buldgen A. Voluntary intake, chemical composition and in vitro digestibility of fresh forages fed to Guinea pigs in periurban rearing systems of Kinshasa (Democratic Republic of Congo). Trop Anim Health Prod 2007; 39(6):419-426.

23. Andrés S, Calleja A, López S, González JS, Rodríguez PL, Giráldez FJ. Prediction of gas production kinetic parameters of forages by chemical composition and near infrared reflectance spectroscopy. Anim Feed Sci Technol 2005; 123-124(1):487-499. 